\title{
Overexpression of $A T G 4 a$ promotes autophagy and proliferation, and inhibits apoptosis in lens epithelial cells via the AMPK and Akt pathways
}

\author{
CHUFAN YAN $^{1}$, JIANGYUE ZHAO ${ }^{1}$, YU QIN ${ }^{1}$, FANGKUN ZHAO ${ }^{1}$, \\ ${\text { LIYANG } \mathrm{JI}^{1} \text { and JINSONG ZHANG }}^{1,2}$ \\ ${ }^{1}$ Department of Ophthalmology, The Fourth Affiliated Hospital of China Medical University, \\ Shenyang, Liaoning 110059; ${ }^{2}$ Cataract Center, Aier Excellent Eye Hospital, \\ Shenyang, Liaoning 110001, P.R. China
}

Received November 12, 2019; Accepted May 15, 2020

DOI: $10.3892 / \mathrm{mmr} .2020 .11205$

\begin{abstract}
Autophagy is a major intracellular degradation system that plays an important role in several biological processes. Although some studies indicate that autophagy may play a role in lens degradation and cataracts formation, its underlying mechanism remains to be elucidated. Autophagy-related gene $4 \mathrm{a}(A T G 4 a)$ cleaves autophagy-related protein 8 (Atg8) near the $\mathrm{C}$ terminus, allowing Atg8 to conjugate with phosphatidylethanolamine via the exposed glycine; although this is pivotal in cancer development, no study has yet linked it to eye diseases. In the present study, the protein expression of ATG4a is significantly upregulated in hydrogen peroxide-treated lens epithelial cells (HLE-B3), indicating that $A T G 4 a$ may play an important role in lens degradation. $A T G 4 a$ was overexpressed using lentivirus in lens epithelial cells to observe the effect of $A T G 4 a$ on various phenotypes by transmission electron microscopy, western blotting, EdU incorporation assay, flow cytometry and in situ cell death detection. The results demonstrated that the overexpression of $A T G 4 a$ could promote autophagy by promoting the adenosine 5'-monophosphate-activated protein kinase pathway and inhibiting the Akt pathway. It also upregulated the proliferation and downregulated the apoptosis of lens epithelial cells. Overall, the present study showed that ATG4a plays a vital role in lens degradation and that it could be a potential target in cataract therapies.
\end{abstract}

Correspondence to: Professor Jinsong Zhang, Department of Ophthalmology, The Fourth Affiliated Hospital of China Medical University, 102 Nanqi Road, Shenyang, Liaoning 110059, P.R. China E-mail: cmuzhangjs@163.com

Abbreviations: ATG4a, autophagy-related gene 4a; PE, phosphatidylethanolamine; TEM, transmission electron microscopy

Key words: apoptosis, autophagy, cataract, lens, proliferation

\section{Introduction}

The lens is an avascular tissue located in front of the vitreous body in the eyes. Light passes through the lens and reaches the retina to form a clear image and therefore the transparency of the lens is important. The lens contains two types of cells, which are lens epithelial cells and lens fiber cells; the organelles in the epithelial cells degrade and differentiate into fiber cells (1). A cataract is one of the most common eye diseases and the leading cause of blindness worldwide (2). Age-related cataracts is the most common type of this disease and it can significantly lower quality of life (3). Perturbation of the lens redox status has been considered as the major cause of age-related cataracts (4). At present, surgical removal of the lens is the primary therapy for this disease. However, there is a lack of effective medical treatment; therefore, several researchers have tried to explain its underlying mechanism in order to delay or eliminate the occurrence of the disease and to identify a therapeutic target $(5,6)$. Hydrogen peroxide $\left(\mathrm{H}_{2} \mathrm{O}_{2}\right)$ elicits reactive oxygen species (ROS) production and ROS increases oxidative stress and crystal protein mutation in lens epithelial cells; therefore, $\mathrm{H}_{2} \mathrm{O}_{2}$ is widely used in building a cataract cell model to simulate the development of an aging lens and the formation of age-related cataract (7).

Autophagy is a major intracellular degradation system, which occurs in almost all cells (8). The dysfunctional cytoplasmic materials, such as mitochondria and endoplasmic reticulum, are sequestered by double-membrane autophagosomes and then delivered into the lysosomes for degradation and recycling (9). Normally, autophagy is divided into the following three types: Macroautophagy, microautophagy and chaperone-mediated autophagy; the first type is the most widely investigated (10). It has been demonstrated that autophagy, especially macroautophagy, may play an important role in the development of cataracts in the lens (11). p62, an autophagy substrate, is downregulated when autophagy is activated (12). By contrast, during autophagy, microtubule-associated protein light chain 3 (LC3)B changes from the soluble form (LC3BI) to the lipid-modified form (LC3BII). Beclin is a critical component of the class III 
phosphatidylinositol 3-kinase complex, which can regulate the autophagy process (13). Therefore, beclin, LC3BII/I and p62 are used as biomarkers to demonstrate the occurrence of autophagy.

Thus far, $>30$ autophagy-related genes have been reported. Among them, autophagy-related gene 4 (ATG4) encodes a cysteine protease that contains four homologs (ATG4A/ATG4B/ATG4C/ATG4D) (14). ATG4a can cleave autophagy-related protein 8 (Atg8) near the $\mathrm{C}$ terminus, allowing Atg8 to conjugate phosphatidylethanolamine (PE) via the exposed glycine. This Atg8-PE system plays an important role in autophagosome formation $(15,16)$.

Although several studies have demonstrated that ATG4 is pivotal in the development of some cancers, such as colorectal cancer, cervical cancer and breast cancer, and it is a promising therapeutic target to treat tumors (17-19), to the best of the authors' knowledge, no research has connected it to lens degradation and cataract formation. As autophagy is a crucial process in lens degradation, the present study explored the biological function of $A T G 4 a$ in lens epithelial cells.

\section{Materials and methods}

Cell culture. The lens epithelial cell line HLE-B3 was purchased from American Type Culture Collection. It was cultured in minimum essential medium (MEM; HyClone; Cytiva) supplemented with $10 \%$ fetal bovine serum (FBS; AusGeneX Pty, Ltd.) and $1 \%$ penicillin-streptomycin solution (HyClone; Cytiva) in a humidified environment of $5 \% \mathrm{CO}_{2}$ at $37^{\circ} \mathrm{C} . \mathrm{H}_{2} \mathrm{O}_{2}(3 \%$; Beijing Solarbio Science \& Technology Co., Ltd.) was diluted with MEM to a concentration of $\sim 200 \mu \mathrm{mol} / 1$. HLE-B3 cells were treated with $200 \mu \mathrm{mol} / 1 \mathrm{H}_{2} \mathrm{O}_{2}$ and termed the $\mathrm{H}_{2} \mathrm{O}_{2}$ group. Untreated HLE-B3 cells were cultured as the control group.

Lentiviral transduction. To overexpress ATG4a, ATG4a sequence was cloned into a lentiviral vector, pLenti-GIIICMV-GFP-2A-Puro vector (cat. no. LV082668; Applied Biological Materials, Inc.). An empty vector was used as a negative control (NC). The sequencing primers of the ATG4a lentiviral vector were as follows: $\mathrm{CMV}$ sequencing primer, 5'-CGC AAA TGG GCG GTA GGC GTG-3'; SV40 reverse sequencing primer, 5'-TAG TCA GCC ATG GGG CGG AGA-3'.

Cells were transferred to 6-well plates at a cell density of $50-60 \%$ and cultured in MEM with $10 \%$ FBS for $24 \mathrm{~h}$. Then, the medium was replaced with Opti-MEM (Thermo Fisher Scientific, Inc.) reduced-serum medium, to which $4.0 \mu \mathrm{g}$ plasmid DNA and $10 \mu 1$ LipoGene $^{\mathrm{TM}} 2000$ Star Transfection Reagent (US Everbright, Inc.) with $0.5 \mathrm{ml}$ Opti-MEM were added; the mixture was incubated for $20 \mathrm{~min}$ at room temperature. The mixture in each plate was mixed with another $2 \mathrm{ml}$ Opti-MEM and incubated under $5 \% \mathrm{CO}_{2}$ in a $37^{\circ} \mathrm{C}$ incubator for $6 \mathrm{~h}$; then, the medium was replaced with complete medium and cultured for another $48 \mathrm{~h}$. Cells transfected with the $A T G 4 a$ and empty vectors were termed the overexpression (OE)-ATG4a and NC groups, respectively. Total proteins were harvested $36 \mathrm{~h}$ after transfection, and other experiments were performed $24 \mathrm{~h}$ after transfection.
Transmission electron microscopy (TEM). Standard TEM was performed for the ultrastructural analysis of the $\mathrm{H}_{2} \mathrm{O}_{2}$, control, OE-ATG $4 a$ and NC groups. Cells were pretreated and fixed with electron microscope-fixing fluid $(2.5 \%$ glutaric dialdehyde solution; Wuhan Servicebio Technology Co., Ltd.) for $2 \mathrm{~h}$ at room temperature and then stored at $4^{\circ} \mathrm{C}$. The cells were then embedded in $1 \%$ agarose and fixed in $1 \%$ osmic acid for $2 \mathrm{~h}$ at room temperature, followed by dehydration with ethanol $(50,70,80,90,95,100 \%)$ and acetone $(100 \%)$ at room temperature for $15 \mathrm{~min}$ each. Cells were then treated with $50 \%$ (2-4 h), 66\% (overnight) and 100\% (5-8 h) epoxy resin at room temperature, followed by $100 \%$ epoxy resin, and heated at $37^{\circ} \mathrm{C}$ overnight and then $60^{\circ} \mathrm{C}$ for $48 \mathrm{~h}$. The agarose-embedded cells were cut into thin sections $(60-80 \mathrm{~nm})$ and stained with $2 \%$ uranyl acetate and $2 \%$ lead citrate for $15 \mathrm{~min}$ at room temperature. Then, the sections were observed at $80 \mathrm{kV}$ by a Hitachi TEM system (HT7700, Hitachi High-Technologies Corporation). Cells and autophagosomes were defined as structures measuring 5.0 and $2.0 \mu \mathrm{m}$, respectively. The results were analyzed by ImageJ version 1.46r (National Institutes of Health) and GraphPad Prism 5 (GraphPad Software, Inc.) software.

Western blotting. Protein expression levels of autophagy biomarkers were determined in the $\mathrm{H}_{2} \mathrm{O}_{2}$, control, OE-ATG4a and $\mathrm{NC}$ groups. The total protein was extracted using the Total Protein Extraction kit for Animal Cultured Cells and Tissues (Invent Biotechnologies, Inc.) on ice and quantified using BCA (Beyotime Institute of Biotechnology). The extracted protein samples were stored at $-80^{\circ} \mathrm{C}$.

Equal amounts of protein $(40 \mu \mathrm{g})$ were electrophoresed via SDS-PAGE on 12\% gels (Beyotime Institute of Biotechnology) and blotted onto polyvinylidene fluoride membranes (EMD Millipore). The membranes were blocked with QuickBlock ${ }^{\mathrm{TM}}$ blocking buffer for western blotting (cat. no. P0252; Beyotime Institute of Biotechnology) for $15 \mathrm{~min}$ at room temperature and then incubated overnight at $4{ }^{\circ} \mathrm{C}$ with the following primary antibodies: Anti-ATG4A (cat. no. ab223374; 1:1,000; Abcam), anti-p62 (cat. no. ab109012; 1:5,000; Abcam), anti-LC3 (cat. no. ab192890; 1:2,000; Abcam), anti-beclin (cat. no. ab207612; 1:2,000; Abcam), anti-Akt (cat. no. YT0178; 1:1,000; ImmunoWay Biotechnology Company), anti-phosphorylated (p-)Akt (cat. no. YP0006; 1:1,000; ImmunoWay Biotechnology Company), anti-adenosine 5'-monophosphate-activated protein kinase (AMPK; cat. no. YT0216; 1:1,000; ImmunoWay Biotechnology Company), anti-p-AMPK (cat. no. YP0575; 1:1,000; ImmunoWay Biotechnology Company), anti-GAPDH (cat. no. ab181602; 1:10,000; Abcam). After washing three times for $10 \mathrm{~min}$ each, the membranes were incubated with the secondary antibodies (cat. no. S0001; 1:2,000; Affinity Biosciences) for $2 \mathrm{~h}$ at room temperature and then washed again in TBS with $0.1 \%$ Tween-20 three times for $10 \mathrm{~min}$ each. The membranes were visualized using the Super ECL Plus kit (US Everbright, Inc.) and developed using a chemiluminescence system (FluorChem FC2 imaging system, ProteinSimple). Signal intensities were visualized by AlphaView software version 3.4.0.0 (ProteinSimple). Data were analyzed with ImageJ version 1.46r (National Institutes of Health) and GraphPad Prism 5 (GraphPad Software, Inc.) software. All experiments were performed in triplicate. 
A
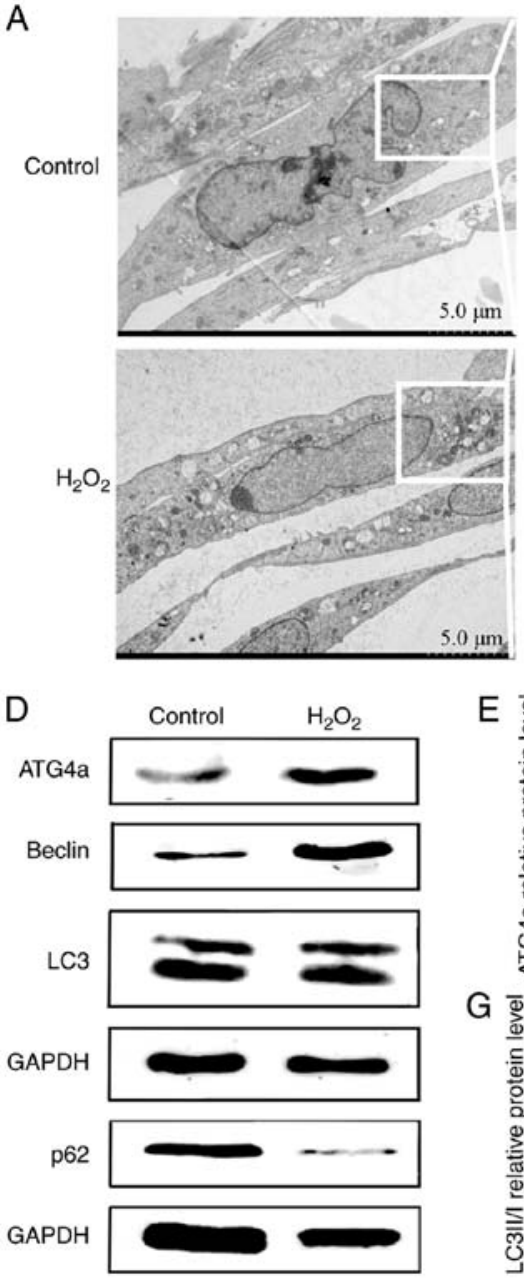
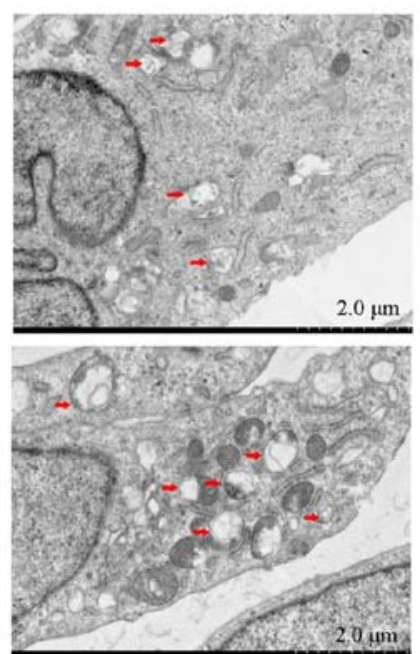

E

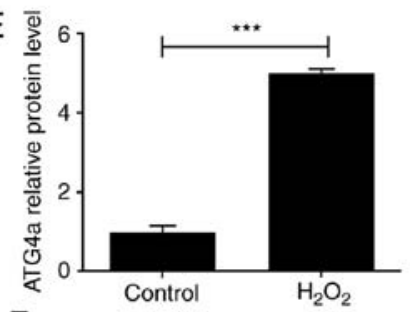

$G$

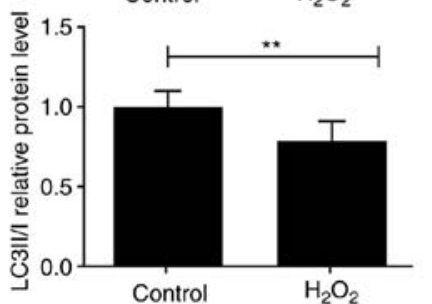

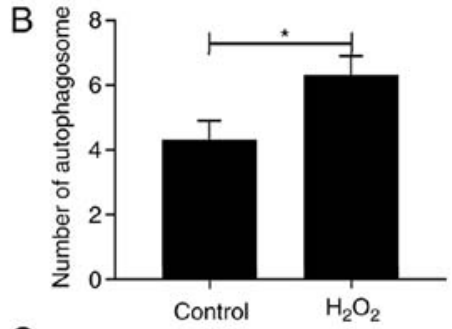
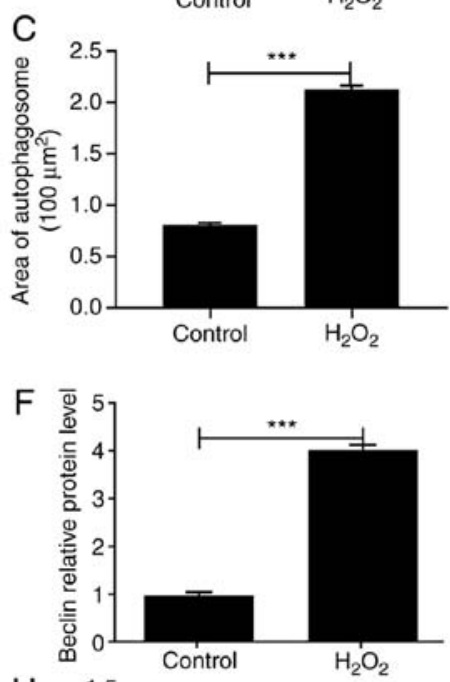

$\mathrm{H}$

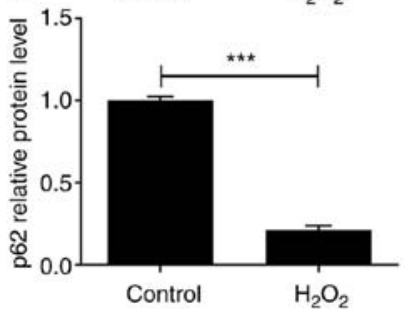

Figure 1. Autophagosomes in lens epithelial cells. (A) Transmission electron micrographs of the autophagosomes (red arrows) in the $\mathrm{H}_{2} \mathrm{O}_{2}$ and control groups. (B) Quantification of the number of autophagosomes in the $\mathrm{H}_{2} \mathrm{O}_{2}$ and control groups. (C) Quantification of the size of autophagosomes in the $\mathrm{H}_{2} \mathrm{O}_{2}$ and control groups. (D) Western blot analysis of the protein expression level of (E) ATG4a and (F) beclin in each group. (G) Quantification of the protein expression ratio of LC3II/I in each group. (H) Quantification of the protein expression level of p62 in each group. ${ }^{*} \mathrm{P}<0.05,{ }^{* *} \mathrm{P}<0.01,{ }^{* * *} \mathrm{P}<0.001$. ATG4a, autophagy-related protein 4 ; LC3, microtubule-associated protein light chain $3 ; \mathrm{H}_{2} \mathrm{O}_{2}$, hydrogen peroxide.

EdU incorporation assay. Cell proliferation rates were analyzed using the EdU incorporation assay in the OE-ATG4a and NC groups. HLE-B3 cells at a density of $80 \%$ were cultured in a 96 -well plate, each group had three replicates. The analysis was performed using the Cell-Light EdU Apollo 488 In Vitro kit (cat. no. C10310-3; Guangzhou RiboBio Co., Ltd.) following the manufacturer's protocol. The percentage of EdU positive cells between the OE-ATG $4 a$ and $\mathrm{NC}$ groups was compared using Image J version 1.46r (National Institutes of Health) and SPSS 23.0 (IBM Corp.) software.

Flow cytometry analysis. Cell apoptosis (early and late apoptosis) in the OE-ATG4a and NC groups was analyzed using a flow cytometer. HLE-B3 cells were cultured in a 6 -well plate and each group had three replicates. The cells were harvested using trypsin without EDTA and washed with PBS twice. The cells were resuspended in $1 \mathrm{X}$ binding buffer at a concentration of $>1 \times 10^{6}$ cells $/ \mathrm{ml}$. Thereafter, $100 \mu \mathrm{l}$ of the solution was transferred into a 5-ml culture tube, to which $5 \mu 1$ FITC Annexin V Apoptosis Detection kit I (BD Biosciences) and $5 \mu \mathrm{l}$ propidium iodide (PI) were added. The mixture was gently vortexed for $15 \mathrm{~min}$ at room temperature in the dark. Subsequently, $400 \mu 1$
$1 \mathrm{X}$ binding buffer was added to each tube and analyzed using BD LSRFortessa and FACSDiva version 6.2 (BD Biosciences) within $1 \mathrm{~h}$. The results were analyzed by ImageJ version $1.46 \mathrm{r}$ (National Institutes of Health) and GraphPad Prism 5 (GraphPad Software, Inc.) software.

In situ cell death detection. Cell death was detected using the In Situ Cell Death Detection kit (Roche Diagnostics) in the OE-ATG $4 a$ and NC groups. HLE-B3 cells at a density of $80 \%$ were cultured in a 96 -well plate at $37^{\circ} \mathrm{C}$, each group had three replicates. In brief, the cells were washed with PBS three times and fixed with $4 \%$ paraformaldehyde in PBS at room temperature for $1 \mathrm{~h}$. The cells were then incubated in permeabilization solution for $2 \mathrm{~min}$ on ice. The TUNEL reaction mixture was prepared and added into each well (50 $\mu \mathrm{l} /$ well); the cells were incubated at $37^{\circ} \mathrm{C}$ in the dark for $1 \mathrm{~h}$. DAPI $(1: 1,000)$ was used to stain the cell nucleus at room temperature for $5 \mathrm{~min}$ in the dark and mounting medium was applied to cells (cat. no. S2110; Beijing Solarbio Science \& Technology Co., Ltd). Images were captured by an Olympus IX71 fluorescence microscope (Olympus Corporation) at magnification, $x 200$. The percentage of dead 
A
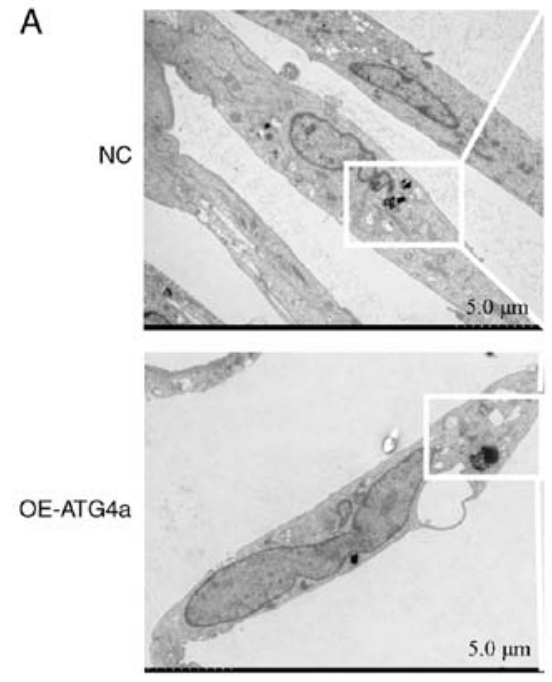

D

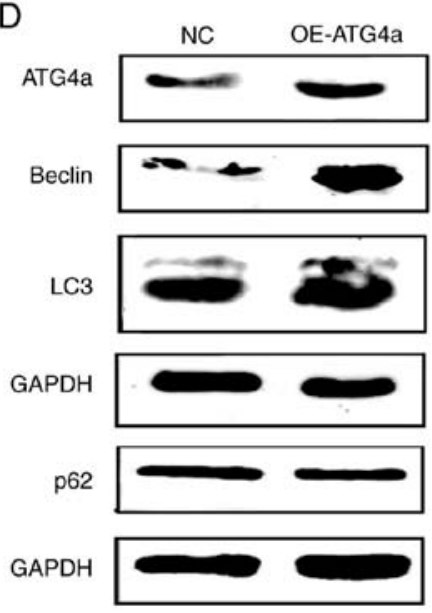

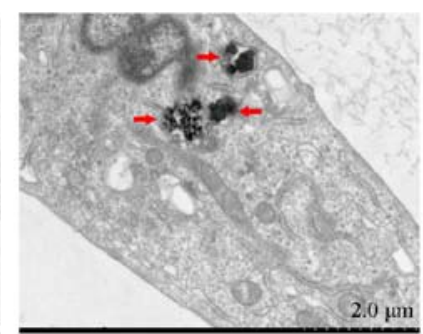

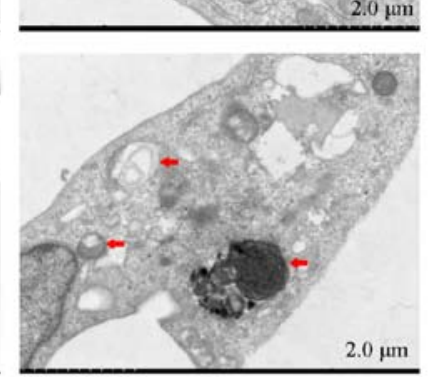

E

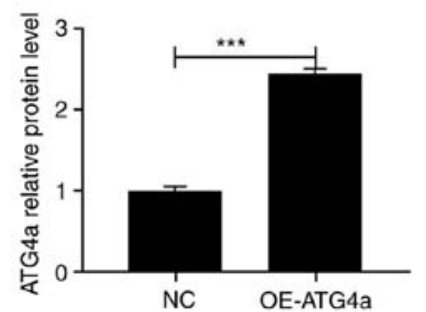

G

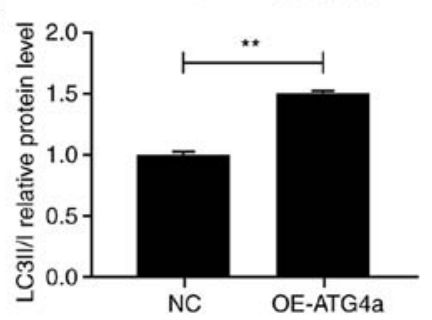

B

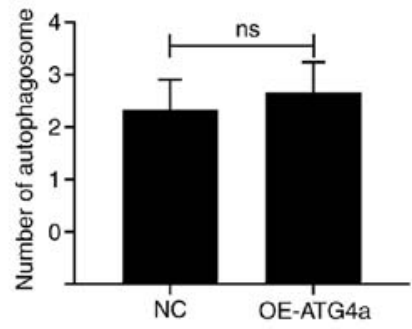

C

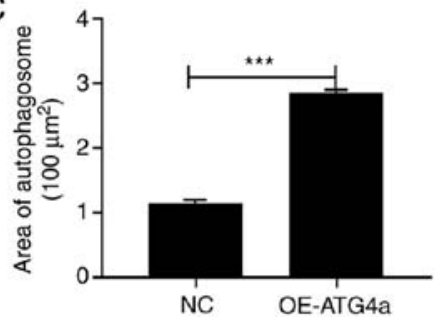

F

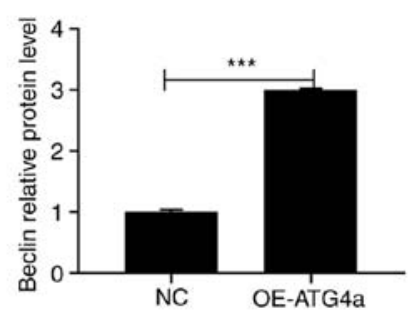

$\mathrm{H}$

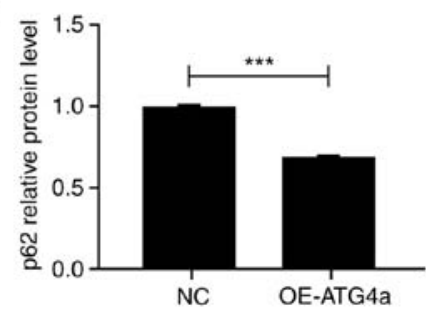

Figure 2. Protein expression level of autophagy biomarkers in lens epithelial cells. (A) Transmission electron micrographs of the autophagosomes (red arrows) in the OE-ATG4a and NC groups. (B) Quantification of the number of autophagosomes in the OE-ATG4a and NC groups. (C) Quantification of the size of autophagosomes in the OE-ATG4a and NC groups. (D) Western blot analysis of the protein expression level of ATG4a, beclin, LC3 and p62 in lens epithelial cells. (E) Quantification of the protein expression level of $A T G 4 a$ and (F) beclin in each group. (G) Quantification of the protein expression ratio of LC3II/I in each group. (H) Quantification of the protein expression level of p62 in each group. ${ }^{* *} \mathrm{P}<0.01,{ }^{* * *} \mathrm{P}<0.001$. NC, negative control; ns, no significance; ATG4a, autophagy-related protein 4; LC3, microtubule-associated protein light chain 3; OE, overexpression group.

cells between the OE-ATG $4 a$ and $\mathrm{NC}$ groups was compared using ImageJ version 1.46r (National Institutes of Health) and SPSS 23.0 (IBM Corp.).

Statistical analysis. All data were analyzed using SPSS version 23 (IBM Corp.) and GraphPad Prism 5 (GraphPad Software, Inc.). The values are presented as mean \pm SD of three independent experiments. The data were analyzed using the independent-samples t-test. $\mathrm{P}<0.05$ was considered to indicate a statistically significant difference.

\section{Results}

$\mathrm{H}_{2} \mathrm{O}_{2}$ can induce autophagy in lens epithelial cells. Autophagy plays an important role in lens degradation and cataract formation (11). To investigate whether $\mathrm{H}_{2} \mathrm{O}_{2}$ can induce autophagy in lens epithelial cells, TEM and western blotting were performed in both $\mathrm{H}_{2} \mathrm{O}_{2}$ and control groups. TEM showed that, compared with the control group, the number $(\mathrm{P}<0.05)$ and size $(\mathrm{P}<0.001)$ of autophagosomes in the $\mathrm{H}_{2} \mathrm{O}_{2}$ group were significantly upregulated, indicating that the rate of autophagy was increased (Fig. 1A-C). The results of western blotting showed that the protein expression of ATG4a was significantly upregulated in the $\mathrm{H}_{2} \mathrm{O}_{2}$ group compared with the control group $(\mathrm{P}<0.001$; Fig. $1 \mathrm{D}$ and $\mathrm{E})$, indicating that this gene might play an important role in autophagy in lens epithelial cells. The protein expression of beclin was significantly upregulated in the $\mathrm{H}_{2} \mathrm{O}_{2}$ group compared with the control group $(\mathrm{P}<0.001$; Fig. 1D and F). By contrast, the protein expression of p62 was significantly downregulated $(\mathrm{P}<0.001$; Fig. $1 \mathrm{D}$ and $\mathrm{H})$. Although the expression of LC3BII/I (P<0.01; Fig.1D and G) did not indicate the upregulation of autophagy, the results of both TEM and western blotting demonstrated the existence of autophagy in $\mathrm{H}_{2} \mathrm{O}_{2}$-induced lens epithelial cells.

Overexpression of ATG4a activates autophagy in lens epithelial cell. To confirm that ATG4a can specifically regulate autophagy, TEM and western blotting were performed in the OE-ATG $4 a$ and NC groups. TEM showed that in the OE-ATG $4 a$ group (Fig. 2A) the amount of autophagosomes 

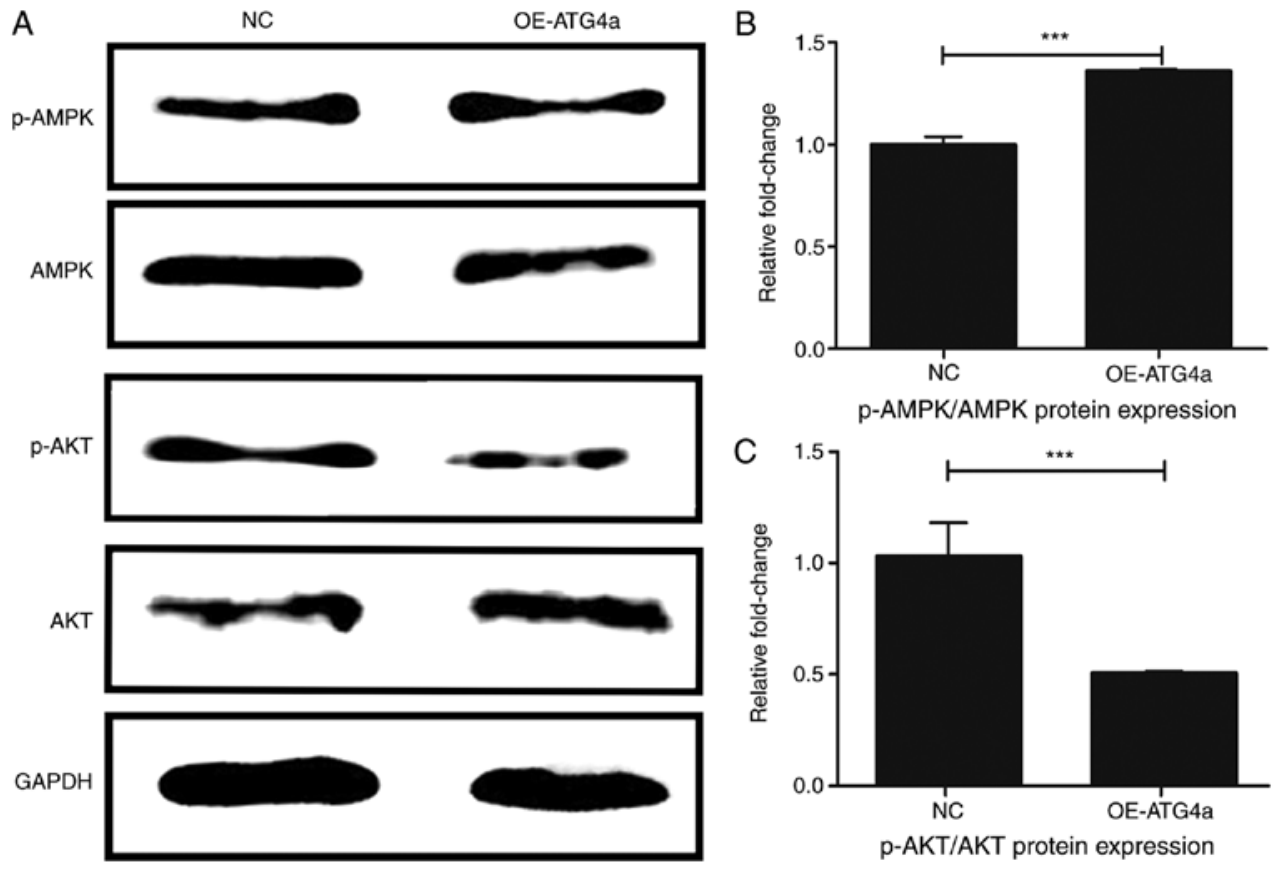

Figure 3. ATG4a promotes the AMPK pathway and inhibits the Akt pathway in lens epithelial cells. (A) Western blot analysis of the protein expression level of p-AMPK, AMPK, p-Akt and Akt in lens epithelial cells. Quantification of the protein expression ratio of (B) p-AMPK/AMPK and (C) p-Akt/Akt in each group. ${ }^{* * *} \mathrm{P}<0.001$. ATG4a, autophagy-related protein 4; AMPK, AMP-activated protein kinase; $\mathrm{p}$-, phosphorylated; OE, overexpression group.

was not significantly different from that in the control group (P>0.05; Fig. 2B), but their size was significantly increased $(\mathrm{P}<0.001 ;$ Fig. $2 \mathrm{C})$. Western blotting confirmed that the protein expression of beclin $(\mathrm{P}<0.001)$ and LC3BII/I $(\mathrm{P}<0.01)$ was significantly upregulated in the OE-ATG4a group compared with the NC group (Fig. 2D, F and G). By contrast, the protein expression of p62 was significantly downregulated $(\mathrm{P}<0.001$; Fig. 2D and $\mathrm{H}$ ), verifying that $A T G 4 a$ can activate autophagy in lens epithelial cells.

ATG4a influences the AMPK and Akt pathways in lens epithelial cells. To investigate the autophagy pathways in which $A T G 4 a$ is involved, western blotting was performed in the OE-ATG4a and NC groups (Fig. 3A). Compared with the NC group, the ratio of protein expression of p-AMPK and AMPK was significantly elevated in the OE-ATG4a group $(\mathrm{P}<0.001$; Fig. 3B), indicating that the overexpression of $A T G 4 a$ significantly activated the phosphorylation of AMPK. By contrast, the trend was reversed for the ratio of p-Akt and Akt $(\mathrm{P}<0.001$; Fig. 3C), indicating that the overexpression of $A T G 4 a$ can inhibit the activation of p-Akt. These results suggested that $A T G 4 a$ may play a role in autophagy by promoting the AMPK pathway and inhibiting the Akt pathway.

ATG4 a promotes proliferation and inhibits apoptosis. To investigate the relationship between ATG4a and cell proliferation and apoptosis, the EdU incorporation assay, FITC/PI double staining tested by flow cytometry and in situ cell death detection assay were performed in the OE-ATG4a and $\mathrm{NC}$ groups. The EdU incorporation assay showed that ATG $4 a$ could significantly promote cell proliferation $(\mathrm{P}<0.05$; Fig. 4A and D). Although the flow cytometry analysis results did not show a significant difference $(\mathrm{P}>0.05)$, it was observed that in the OE-ATG4a group, the apoptosis rate of lens epithelial cells was lower compared with that in the NC group, indicating that $A T G 4 a$ may inhibit apoptosis (Fig. 4B and E). To further confirm this finding, a cell death detection assay was performed in the OE-ATG4 $a$ and NC groups. The results showed that, in the OE-ATG4a group, the percentage of TUNEL positive cells was lower compared with that in the NC group $(\mathrm{P}<0.001)$ and the difference was significant, indicating that $A T G 4 a$ can inhibit apoptosis (Fig. $4 \mathrm{C}$ and F).

\section{Discussion}

The present study for the first time, to the best of the authors' knowledge, demonstrated that $A T G 4 a$-mediated autophagy played an important role in the proliferation and apoptosis of lens epithelial cells, and that this might occur via the AMPK and Akt pathways.

$\mathrm{H}_{2} \mathrm{O}_{2}$-induced lens epithelial cells have been widely used as a model for lens degradation and cataract formation, according to previous studies, $200 \mu \mathrm{mol} / 1$ was chosen as the concentration $(20,21)$. In the present study, this model was used to simulate the degradation of the lens in the eyes. TEM showed that the number and size of autophagosomes in the $\mathrm{H}_{2} \mathrm{O}_{2}$ group were significantly higher compared with those in the control group. The western blot analysis further validated the results; the protein expression of beclin was significantly increased and the protein expression of p62 was decreased after $\mathrm{H}_{2} \mathrm{O}_{2}$ treatment, suggesting that autophagy was significantly upregulated in $\mathrm{H}_{2} \mathrm{O}_{2}$-treated lens epithelial cells. Furthermore, western blotting showed that the protein expression of ATG4a was upregulated in $\mathrm{H}_{2} \mathrm{O}_{2}$-treated lens epithelial cells.

Briefly, ATG4a is an enzyme that can cleave LC3I to produce LC3II; It plays an important role in the formation of autophagic vesicle membranes (12). ATG4a is also the only protease encoded by the autophagy-related genes (22). The 
A

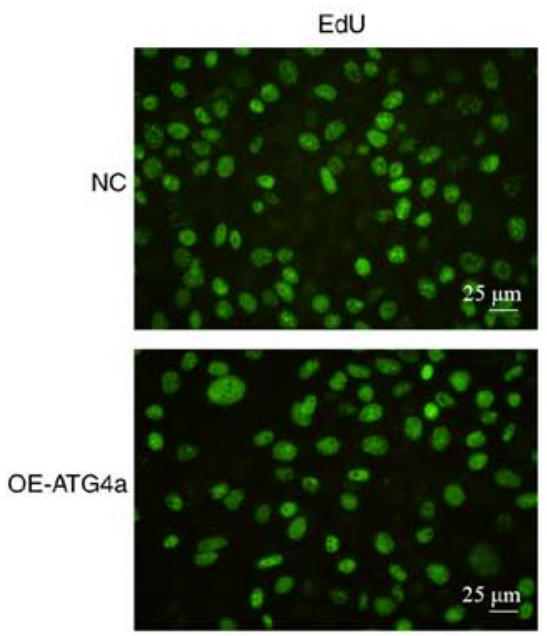

B

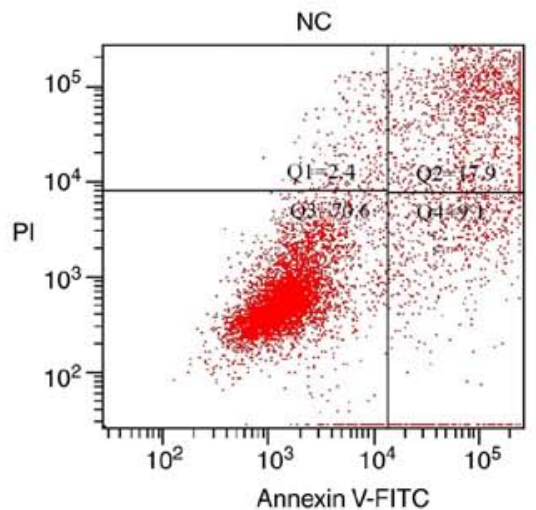

Hoechst
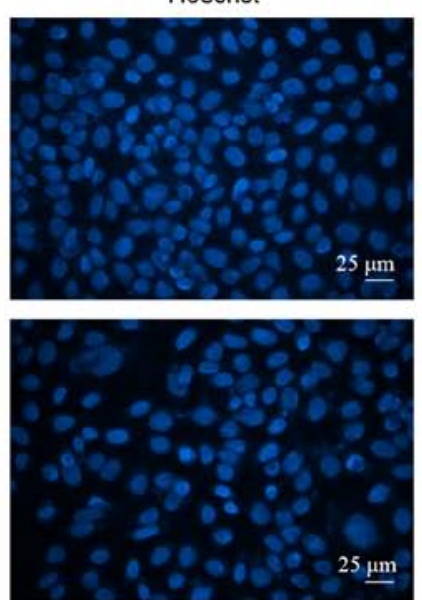

Merge
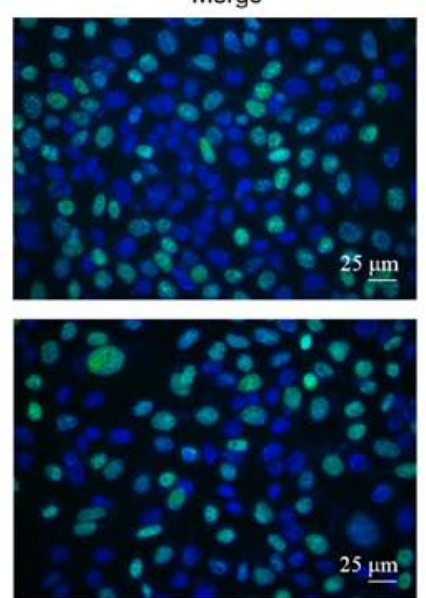

OE-ATG4a

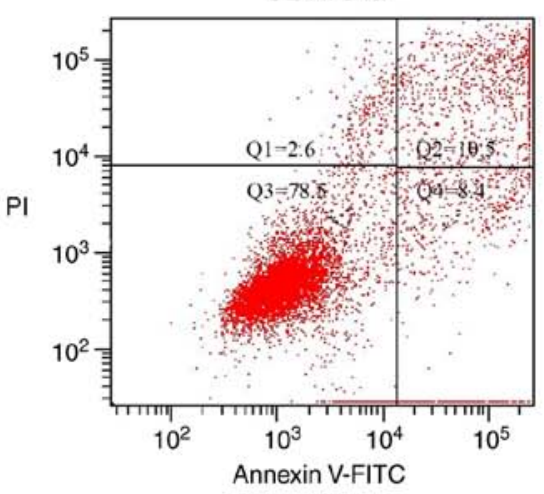

C

TUNEL
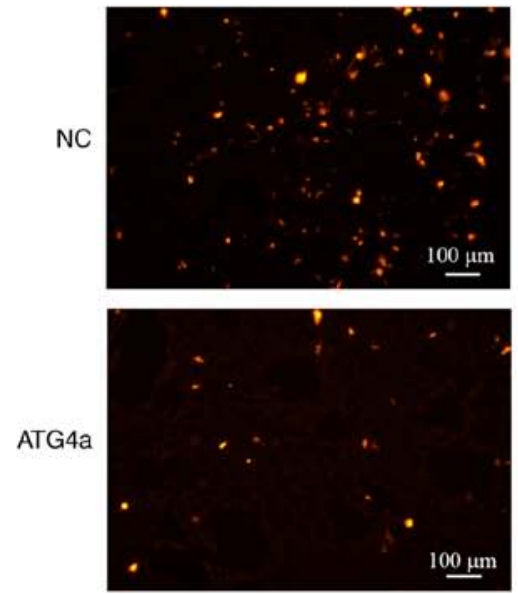

DAPI
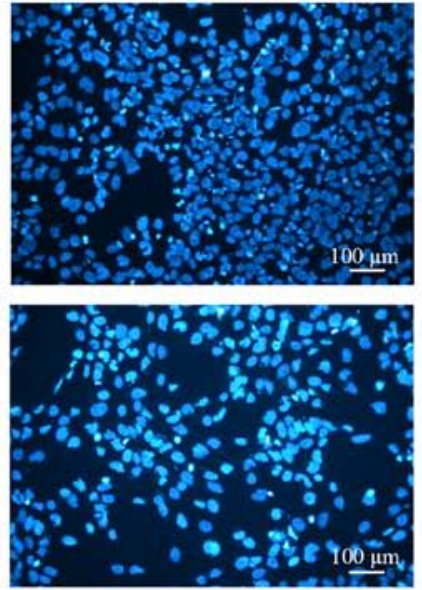
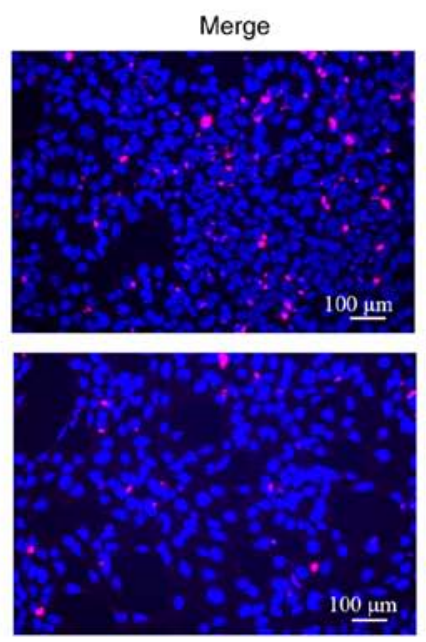

$\mathrm{F}$

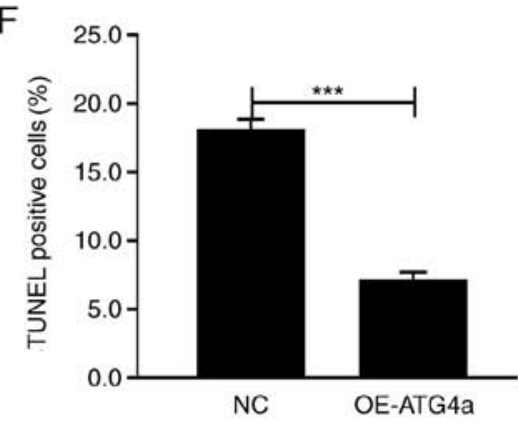

Figure 4. ATG4a promotes proliferation and inhibits the apoptosis of lens epithelial cells. (A) EdU incorporation assay in the OE-ATG4a and NC groups. (B) Flow cytometry results of Annexin V-FITC/PI staining in the OE-ATG4a and NC groups. (C) Cell death assay results of TUNEL staining in the OE-ATG4a and NC groups. (D) Quantification of EdU-positive cells between the OE-ATG4a and NC groups. (E) Quantification of Annexin V-FITC/PI staining between the OE-ATG4a and NC groups. (F) Quantification of TUNEL positive cells between the $\mathrm{OE}-A T G 4 a$ and $\mathrm{NC}$ groups. ${ }^{*} \mathrm{P}<0.05$, ${ }^{* * *} \mathrm{P}<0.001$. ATG4a, autophagy-related protein 4; ns, no significance; NC, negative control; OE, overexpression group; PI, propidium iodide. 
upregulation of ATG4a in $\mathrm{H}_{2} \mathrm{O}_{2}$-treated lens epithelial cells indicated that $A T G 4 a$ may play a key role in lens degradation.

Previous studies have demonstrated that $A T G 4 a$ can promote metastasis of tumor cells (23) and can be used as a target in chemotherapy of cancer (24). To explore the biological functions of $A T G 4 a$ in lens epithelial cells, a model of $A T G 4 a$ overexpression was constructed. TEM demonstrated that, compared with the NC group, the size of autophagosomes increased in the OE-ATG4a group. Several techniques including western blotting, immunofluorescence assay and a dual-fluorescence mRFP-eGFP-LC3 system can evaluate the function of effector proteins and autophagy flux $(25,26)$. In the present study, western blotting demonstrated that the protein expression of beclin and LC3II/I was upregulated and that of p62 was downregulated in the OE-ATG $4 a$ group. TEM is considered one of the main and most important methods for the detection of autophagy $(27,28)$. Furthermore, beclin, LC3II/I and p62 are routinely used as biomarkers to measure the rate and occurrence of autophagy (29-32). The results indicated that $A T G 4 a$ could activate autophagy in lens epithelial cells.

The Akt and AMPK signaling pathways are important pathways in the autophagy process. Normally, Akt activates mammalian target of rapamycin complex 1 , which is a negative regulator of autophagy, by inhibiting the tuberous sclerosis complex 1/2 (TSC1/TSC2) protein complex and thus inhibiting autophagy (33). In a previous study, the ratio of p-Akt/Akt in H9C2 cells was significantly reduced following exposure to $\mathrm{H}_{2} \mathrm{O}_{2}$ and triggered autophagy, indicating that the Akt pathway at least partly modulates autophagy (34). AMPK activation leads to the phosphorylation and activation of TSC1/2 and inhibition of mTOR; thus indicating that it is a positive regulator of autophagy (35). In the present study, the ratio of p-AMPK/AMPK was significantly increased and the ratio of p-Akt/Akt was decreased in the OE-ATG4a group compared with the NC group, indicating that these two pathways might be modulated by $A T G 4 a$ in lens epithelial cells, but the exact mechanism needs further research. Nevertheless, the activation and inhibition of these two autophagy pathways further demonstrated that $A T G 4 a$ can promote autophagy in lens epithelial cells.

Increasing evidence has demonstrated that cell proliferation and apoptosis are affected by autophagy $(8,36,37)$ Studies have demonstrated that autophagy can promote cell growth $(38,39)$, but the relationship between apoptosis and autophagy is complex and often appears contradictory $(36,40)$. Normally, autophagy can maintain cell homeostasis under stressful conditions and prevent cell death (8), but in some neurodegenerative diseases, such as Alzheimer's disease, it can also be a pathogenic factor (37). Therefore, elucidating the exact functions of autophagy genes will improve our knowledge on how to protect or induce cell death. The present study demonstrated that ATG $4 a$ had a positive effect on cell proliferation, using the EdU incorporation assay. As for apoptosis, although the FITC/PI staining assay did not show a statistical difference between the two groups, the in situ cell death detection assay showed that the apoptosis rate in the OE-ATG4a group was significantly downregulated compared with the NC group, suggesting that $A T G 4 A$ could inhibit apoptosis in lens epithelial cells.
Taken together, the present study demonstrated that ATG $4 a$ could induce autophagy in lens epithelial cells and that this might activate the AMPK and inhibit Akt pathways. Furthermore, $A T G 4 a$ increased cell proliferation and decreased apoptosis, indicating that $A T G 4 a$ plays an important role in lens degradation and cataract formation.

\section{Acknowledgements}

Not applicable.

\section{Funding}

This work was supported by the National Natural Science Foundation of China (grant no. 81870644).

\section{Availability of data and materials}

The datasets used and/or analyzed during the current study are available from the corresponding author on reasonable request.

\section{Authors' contributions}

CY performed all experiments and wrote the manuscript. JZ, YQ, FZ and LJ analyzed the experimental data. JZ designed the present study and wrote the manuscript. All authors read and approved the final manuscript.

\section{Ethics approval and consent to participate}

Not applicable.

\section{Patient consent for publication}

Not applicable.

\section{Competing interests}

The authors declare that they have no competing interests.

\section{References}

1. Augusteyn RC: Growth of the lens: In vitro observations. Clin Exp Optom 91: 226-239, 2008

2. Pascolini D and Mariotti SP: Global estimates of visual impairment: 2010. Br J Ophthalmol 96: 614-618, 2012.

3. Flaxman SR, Bourne RRA, Resnikoff S, Ackland P, Braithwaite T, Cicinelli MV, Das A, Jonas JB, Keeffe J, Kempen JH, et al; Vision Loss Expert Group of the Global Burden of Disease Study: Global causes of blindness and distance vision impairment 1990-2020: A systematic review and meta-analysis. Lancet Glob Health 5: e1221-e1234, 2017.

4. Zhao WJ and Yan YB: Increasing susceptibility to oxidative stress by cataract-causing crystallin mutations. Int $\mathrm{J}$ Biol Macromol 108: 665-673, 2018.

5. Feng H, Yang Z, Bai X, Yang M, Fang Y, Zhang X, Guo Q and Ning H: Therapeutic potential of a dual mTORC1/2 inhibitor for the prevention of posterior capsule opacification: An in vitro study. Int J Mol Med 41: 2099-2107, 2018.

6. Crooke A, Huete-Toral F, Colligris B and Pintor J: The role and therapeutic potential of melatonin in age-related ocular diseases. J Pineal Res 63: e12430, 2017.

7. Jin X, Jin H, Shi Y, Guo Y and Zhang H: Long Non-Coding RNA KCNQ1OT1 promotes cataractogenesis via miR-214 and activation of the Caspase-1 pathway. Cell Physiol Biochem 42: 295-305, 2017. 
8. Choi AM, Ryter SW and Levine B: Autophagy in human health and disease. N Engl J Med 368: 651-662, 2013.

9. Ni Z, Gong Y, Dai X, Ding W, Wang B, Gong H, Qin L, Cheng P, Li S, Lian J, et al: AU4S: A novel synthetic peptide to measure the activity of ATG4 in living cells. Autophagy 11: 403-415, 2015.

10. Wang RC and Levine B: Autophagy in cellular growth control FEBS Lett 584: 1417-1426, 2010.

11. Morishita $\mathrm{H}$ and Mizushima N: Autophagy in the lens. Exp Eye Res 144: 22-28, 2016

12. Lv W, Sui L, Yan X, Xie H, Jiang L, Geng C, Li Q, Yao X, Kong Y and Cao J: ROS-dependent Atg4 upregulation mediated autophagy plays an important role in Cd-induced proliferation and invasion in A549 cells. Chem Biol Interact 279: 136-144, 2018.

13. He R, Peng J, Yuan P, Xu F and Wei W: Divergent roles of BECN1 in LC3 lipidation and autophagosomal function. Autophagy 11 740-747, 2015

14. Li M, Hou Y, Wang J, Chen X, Shao ZM and Yin XM: Kinetics comparisons of mammalian Atg4 homologues indicate selective preferences toward diverse Atg8 substrates. J Biol Chem 286: 7327-7338, 2011

15. Nakatogawa H, Ichimura $Y$ and Ohsumi Y: Atg8, a ubiquitin-like protein required for autophagosome formation, mediates membrane tethering and hemifusion. Cell 130: 165-178, 2007.

16. Scherz-Shouval R, Shvets E, Fass E, Shorer H, Gil L and Elazar Z: Reactive oxygen species are essential for autophagy and specifically regulate the activity of Atg4. EMBO J 26 : 1749-1760, 2007.

17. Gil J, Ramsey D, Pawlowski P, Szmida E, Leszczynski P, Bebenek $M$ and Sasiadek MM: The influence of tumor microenvironment on ATG4D gene expression in colorectal cancer patients. Med Oncol 35: 159-167, 2018.

18. Mao JJ, Wu LX, Wang W, Ye YY, Yang J, Chen H, Yang QF, Zhang XY, Wang B and Chen WX: Nucleotide variation in ATG4A and susceptibility to cervical cancer in Southwestern Chinese women. Oncol Lett 15: 2992-3000, 2018.

19. Antonelli M, Strappazzon F, Arisi I, Brandi R, D'Onofrio M, Sambucci M, Manic G, Vitale I, Barilà D and Stagni V: ATM kinase sustains breast cancer stem-like cells by promoting ATG4C expression and autophagy. Oncotarget 8: 21692-21709, 2017.

20. Zhou W, Xu J, Wang C, Shi D and Yan Q: miR-23b-3p regulates apoptosis and autophagy via suppressing SIRT1 in lens epithelial cells. J Cell Biochem 120: 19635-19646, 2019.

21. De-Qian K, Yue L, Li L and Guangying Z: Downregulation of Smac attenuates $\mathrm{H}_{2} \mathrm{O}_{2}$-induced apoptosis via endoplasmic reticulum stress in human lens epithelial cells. Medicine (Baltimore) 96: e7419, 2017.

22. Fernández ÁF and López-Otín C: The functional and pathologic relevance of autophagy proteases. J Clin Invest 125: 33-41, 2015.

23. Yang SW, Ping YF, Jiang YX, Luo X, Zhang X, Bian XW and Yu PW: ATG4A promotes tumor metastasis by inducing the epithelial-mesenchymal transition and stem-like properties in gastric cells. Oncotarget 7: 39279-39292, 2016.

24. Liu PF, Tsai KL, Hsu CJ, Tsai WL, Cheng JS, Chang HW, Shiau CW, Goan YG, Tseng $\mathrm{HH}$, Wu CH, et al: Drug repurposing screening identifies tioconazole as an ATG4 inhibitor that suppresses autophagy and sensitizes cancer cells to chemotherapy. Theranostics 8: 830-845, 2018.

25. Lee JH, Rao MV, Yang DS, Stavrides P, Im E, Pensalfini A, Huo C, Sarkar P, Yoshimori T and Nixon RA: Transgenic expression of a ratiometric autophagy probe specifically in neurons enables the interrogation of brain autophagy in vivo. Autophagy 15: 543-557, 2019.
26. Sun Y, Huang YH, Huang FY, Mei WL, Liu Q, Wang CC, Lin YY, Huang C, Li YN, Dai HF, et al: 3'-epi-12 $\beta$-hydroxyfroside, a new cardenolide, induces cytoprotective autophagy via blocking the Hsp90/Akt/mTOR axis in lung cancer cells. Theranostics 8 2044-2060, 2018.

27. Biazik J, Vihinen H, Anwar T, Jokitalo E and Eskelinen EL: The versatile electron microscope: An ultrastructural overview of autophagy. Methods 75: 44-53, 2015.

28. Klionsky DJ, Abdelmohsen K, Abe A, Abedin MJ, Abeliovich H, Arozena AA, Adachi H, Adams CM, Adams PD, Adeli K, et al: Guidelines for the use and interpretation of assays for monitoring autophagy (3rd edition). Autophagy 12: 1-222, 2016.

29. Runwal G, Stamatakou E, Siddiqi FH, Puri C, Zhu Y and Rubinsztein DC: LC3-positive structures are prominent in autophagy-deficient cells. Sci Rep 9: 10147, 2019.

30. Bortnik S and Gorski SM: Clinical applications of autophagy proteins in cancer: From potential targets to biomarkers. Int J Mol Sci 18: E1496, 2017.

31. Fan J, Yang X, Li J, Shu Z, Dai J, Liu X, Li B, Jia S, Kou X, Yang Y, et al: Spermidine coupled with exercise rescues skeletal muscle atrophy from D-gal-induced aging rats through enhanced autophagy and reduced apoptosis via AMPK-FOXO3a signal pathway. Oncotarget 8: 17475-17490, 2017.

32. Wang S, Ji LY, Li L and Li JM: Oxidative stress, autophagy and pyroptosis in the neovascularization of oxygen-induced retinopathy in mice. Mol Med Rep 19: 927-934, 2019.

33. Yang Z and Klionsky DJ: Mammalian autophagy: Core molecular machinery and signaling regulation. Curr Opin Cell Biol 22: 124-131, 2010.

34. Liu L, Jin X,Hu CF, Li R, Zhou Z and Shen CX: Exosomes derived from mesenchymal stem cells rescue myocardial ischaemia/reperfusion injury by inducing cardiomyocyte autophagy via AMPK and Akt pathways. Cell Physiol Biochem 43: 52-68, 2017.

35. Zhao M, Sun L, Yu XJ, Miao Y, Liu JJ, Wang H, Ren J and Zang WJ: Acetylcholine mediates AMPK-dependent autophagic cytoprotection in $\mathrm{H} 9 \mathrm{c} 2$ cells during hypoxia/reoxygenation injury. Cell Physiol Biochem 32: 601-613, 2013.

36. Booth LA, Tavallai S, Hamed HA, Cruickshanks N and Dent P. The role of cell signalling in the crosstalk between autophagy and apoptosis. Cell Signal 26: 549-555, 2014.

37. Levine B and Kroemer G: Autophagy in the pathogenesis of disease. Cell 132: 27-42, 2008.

38. Katheder NS, Khezri R, O'Farrell F, Schultz SW, Jain A, Rahman MM, Schink KO, Theodossiou TA, Johansen T, Juhász G, et al: Microenvironmental autophagy promotes tumour growth. Nature 541: 417-420, 2017.

39. Kimmelman AC and White E: Autophagy and tumor metabolism. Cell Metab 25: 1037-1043, 2017.

40. Wang $\mathrm{H}$ and Zhang G: Activation of CaMKK $\beta$-AMPK-mTOR pathway is required for autophagy induction by $\beta, \beta$-dimethylacrylshikonin against lung adenocarcinoma cells. Biochem Biophys Res Commun 517: 477-483, 2019.

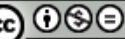

This work is licensed under a Creative Commons Attribution-NonCommercial-NoDerivatives 4.0 International (CC BY-NC-ND 4.0) License. 